\title{
Imaging of the trachea
}

\section{Jo-Anne O. Shepard, Efren J. Flores, Gerald F. Abbott}

Division of Thoracic Imaging, Department of Radiology, Massachusetts General Hospital, Harvard Medical School, Boston, MA, USA

Correspondence to: Jo-Anne O. Shepard, MD. Thoracic Imaging, Department of Radiology, Massachusetts General Hospital, Founders 202, 55 Fruit Street, Boston, MA 02114, USA. Email: jshepard@partners.org.

\begin{abstract}
Numerous benign and malignant tracheal diseases may affect the trachea primarily and secondarily. While the posterior anterior (PA) and lateral chest radiograph is the conventional study for initial evaluation of the trachea and central airways, findings may not always be apparent on conventional radiographs, and further evaluation with cross sectional imaging is usually necessary. Computed tomography (CT) is the imaging modality of choice for imaging the trachea and bronchi. Familiarity with the imaging appearances of the normal and diseased trachea will enhance diagnostic evaluation.
\end{abstract}

Keywords: Trachea; tracheal stenosis; tracheal tumor; tracheomalacia

Submitted Jan 09, 2018. Accepted for publication Feb 27, 2018.

doi: 10.21037/acs.2018.03.09

View this article at: http://dx.doi.org/10.21037/acs.2018.03.09

\section{Introduction}

The trachea extends from the cricoid cartilage to the carina and usually measures $10-12 \mathrm{~cm}$ in length. The extra thoracic trachea is $(2-4 \mathrm{~cm}$ in length) and transitions to the intrathoracic trachea $(6-9 \mathrm{~cm}$ in length) as it passes posterior to the manubrial notch. Cartilaginous $\mathrm{C}$-shaped rings (1-4) form the anterior and lateral walls of the trachea. The posterior "membranous" wall of the trachea is formed by the posterior tracheal membrane. Multiple benign and malignant conditions may alter the normal appearance of the trachea. This review focuses on the imaging appearance of various benign and malignant disease entities that can affect the trachea $(1,5,6)$.

\section{Imaging technique}

\section{Radiography}

The posterior anterior (PA) and lateral chest radiograph is the conventional study for initial evaluation of the trachea and central airways. On chest radiographs, the distal cervical trachea, intrathoracic trachea and main bronchi are visible; however, overlying mediastinal structures often obscure intrathoracic tracheobronchial abnormalities. Bilateral oblique chest radiographs can improve tracheal evaluation by rotating the spine and mediastinal structures away from the trachea. Visualization of tracheal abnormalities can also be improved by high kilovoltage technique $(140 \mathrm{kV})$. Evaluation of the trachea and central airways should be carefully performed on all patients presenting with symptoms of stridor, wheezing, "adult-onset" asthma, hemoptysis or recurrent pneumonia. Findings may not always be apparent on conventional radiographs and further evaluation with cross sectional imaging is usually necessary.

\section{Computed tomography (CT)}

CT is the imaging modality of choice for imaging the trachea and bronchi for several reasons (2-4). CT can directly demonstrate the normal anatomy and appearance of the airways (Figure 1). It can specifically evaluate the adjacent mediastinal structures, mediastinal invasion by tumors or airway compression by adjacent mediastinal masses. CT also provides important morphologic information regarding vascular enhancement and tissue characteristics such as calcification and fatty components of a lesion.

Current CT scanners can scan the entire chest in a few seconds. Images can be quickly reconstructed with thin sections in multiple planes including axial, coronal and sagittal planes. Off-axis coronal images are useful in imaging the trachea in a single plane. Advances in imaging 

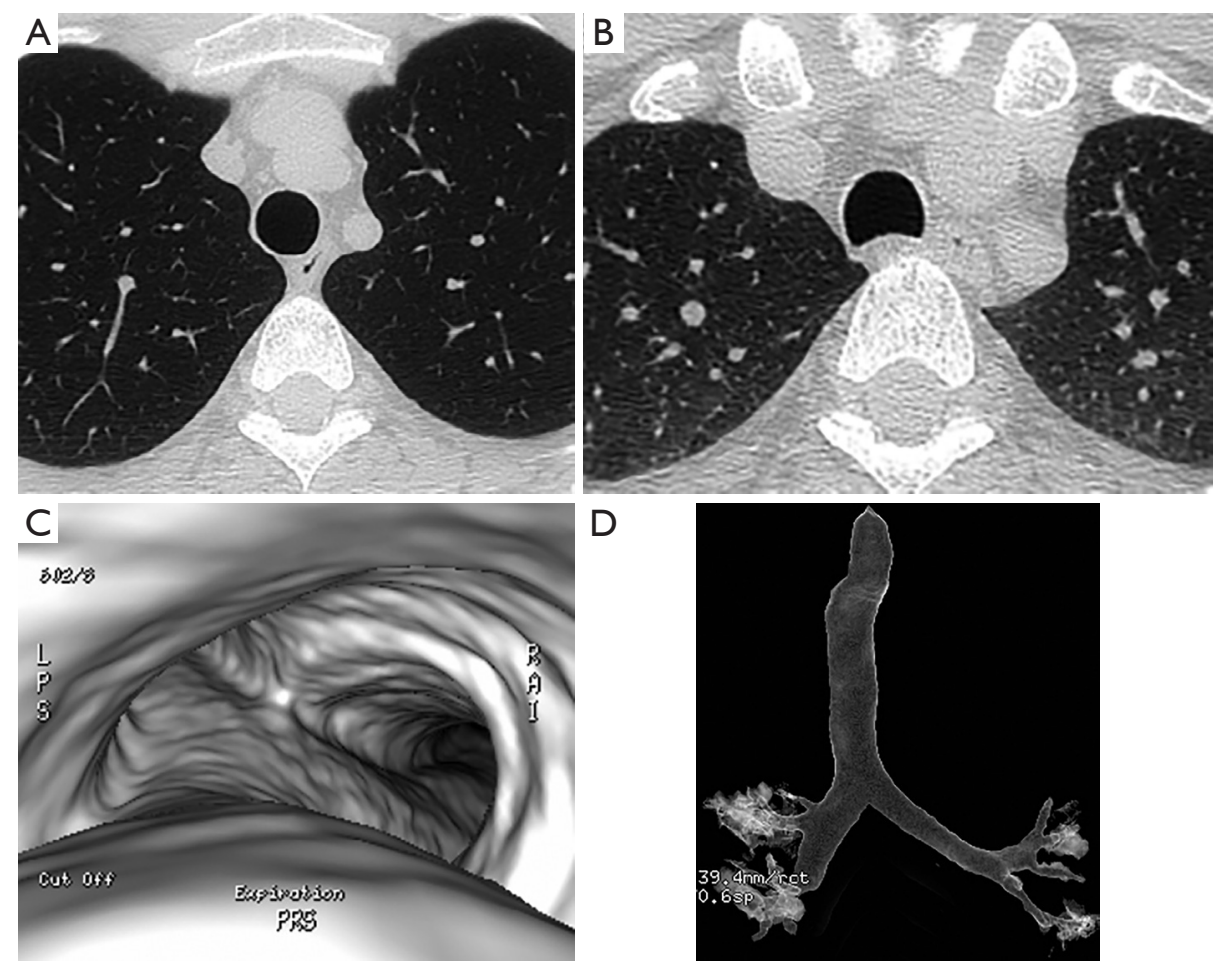

Figure 1 Normal trachea. Paired inspiratory (A) and expiratory (B) axial CT images in lung windows demonstrate a thin smooth tracheal wall. On inspiration, the trachea has a normal circular configuration, but on expiration the posterior membranous wall becomes concave in configuration. Normally the tracheal lumen may decrease by $30 \%$ on expiration. Note that on expiration, the lung density also increases. A virtual bronchoscopic image (C) of the normal trachea at the level of the carina depicts the trachea from the perspective of bronchoscopy. The right main bronchus is seen on the right side of the image and the left main bronchus is seen on the left side of the image. The anterior trachea wall is seen at the top of the image and the posterior tracheal wall is seen at the bottom of the image. Note the slight indentation of the posterior membranous wall and the cartilaginous rings of the trachea. A 3D surface rendering of the trachea (D) demonstrates the normal trachea, main and lobar bronchi.

including volumetric imaging and dynamic imaging have enhanced diagnostic capabilities. Forced expiratory imaging or imaging performed during coughing can improve the evaluation of tracheomalacia (Figure 1). The most commonly employed 3D imaging techniques of the airways include external volume rendering and internal volume rendering, i.e., surface rendering techniques and virtual bronchoscopy (Figure 1). While these additional images do not replace axial imaging they can improve the understanding of the craniocaudal extent of disease and help in planning for other diagnostic interventions.

\section{Magnetic resonance imaging (MRI)}

While the trachea and main bronchi are generally well- visualized on MRI, MRI imaging is usually less reproducible than CT and MRI takes longer to perform. One of the major advantages of MRI is the lack of ionizing radiation which is appealing in evaluating young children and adolescents who may require frequent imaging of the trachea. MRI is also advantageous in imaging tracheal compression or invasion by mediastinal masses or vascular rings or other vascular anomalies that may compress the trachea.

\section{Fluorodeoxyglucose (FDG) positron emission tomography (PET)/CT}

FDG PET/CT is reserved for staging patients with tracheobronchial malignancies. 

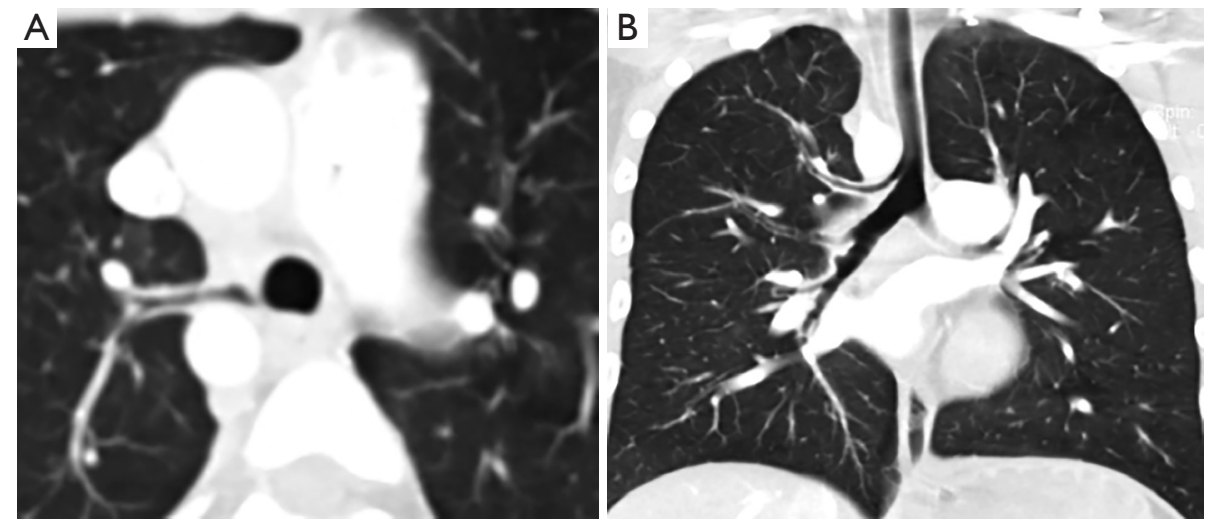

Figure 2 Tracheal "pig" bronchus. Axial CT (A) and coronal CT (B) images in lung windows demonstrate a tracheal bronchus arising from the right lateral wall of the distal trachea proximal to the take off the right upper lobe bronchus. In this case, a displaced bronchus supplies a portion of the right upper lobe.

\section{Tracheal anomalies}

\section{Tracheal bronchus}

A tracheal bronchus or "pig" bronchus is the most commonly seen bronchial anomaly (7). It arises from the right lateral tracheal wall usually within $2 \mathrm{~cm}$ of the carina. A left tracheal bronchus also occurs much less commonly. There are four different anatomic types: (I) the most common is the displaced bronchus in which one or two of the upper lobe segments are aerated by the tracheal bronchus; (II) a supernumerary accessory bronchus which arises from the right lateral tracheal wall in addition to the normal right upper lobe bronchi; (III) a right upper lobe bronchus with three normal bronchial segments that arises above the carina and (IV) a rudimentary tracheal bronchus presenting as a blind out pouching from the right lateral tracheal wall. The presence of a tracheal bronchus is usually an incidental finding but does have implications for endotracheal tube placement and lung resections (Figure 2).

\section{Cardiac bronchus}

A cardiac bronchus is an uncommon bronchial anomaly that arises as a small bronchus from the medial aspect of the right main bronchus. It may present as a blind out pouching of 1-2 cm in length. Occasionally, it may lead to a small vestige of lung in that may have a pleural investiture. Rarely, patients with cardiac bronchi may present with hemoptysis.

\section{Tracheal widening}

\section{Tracheal diverticula}

Tracheal diverticula or cysts are small air filled outpouchings from the tracheal wall resulting from focal herniation of tracheal mucosa through the tracheal wall (8). They can be congenital or acquired and are generally seen in the right paratracheal region $4-5 \mathrm{~cm}$ below the level of the vocal cords near the thoracic inlet. The acquired diverticula are thought to be associated with chronic cough and increased intraluminal pressure and are often seen in patients with chronic obstructive airways diseases. Tracheal diverticula are usually discovered incidentally on CT and are not usually detected on standard radiographs due to their small size (Figure 3).

\section{Tracheobronchomegaly/Mounier-Kuhn syndrome}

Mounier-Kuhn syndrome is a congenital condition with an underlying defect in smooth muscle and elastic tissue $(9,10)$. It is associated with recurrent respiratory infections and occurs more frequently in males and is usually diagnosed in the third to fourth decades of life. There is diffuse dilatation of the trachea, main and lobar bronchi with abrupt transition to normal appearance bronchi at the segmental level. Characteristically the mucosa protrudes through the trachealis muscle between the cartilaginous rings. A resultant scalloped or corrugated appearance of the tracheal and main bronchial walls is seen on radiographs or CT. Recurrent infections often lead to bilateral bronchiectasis. 

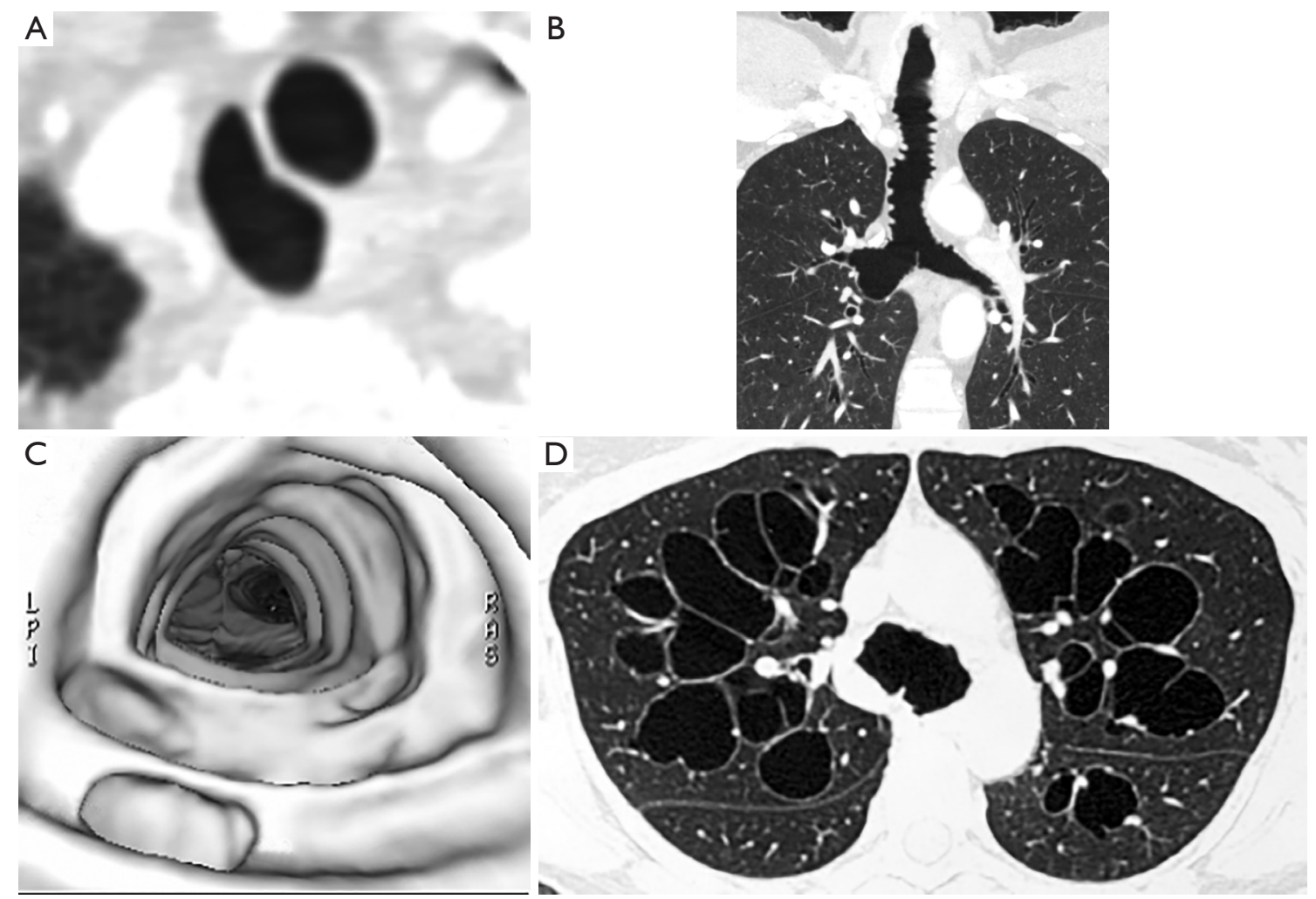

Figure 3 Tracheal diverticulum and tracheobronchomegaly, Mounier-Kuhn syndrome. In a patient with a tracheal diverticulum, an axial CT image (A) of the trachea just below the thoracic inlet demonstrates an air-filled cystic structure arising from the right lateral wall of the trachea. In a patient with Mounier-Kuhn syndrome (B-D), a coronal CT image (B) reveals diffuse tracheobronchomegaly with typical scalloped appearance of the tracheal and main bronchial walls. A virtual bronchoscopic image (C) shows the dilated trachea and the outpouchings of the wall manifesting as "diverticula". An axial CT image in lung windows (D) demonstrates varicose and cystic bronchiectasis of the central subsegmental bronchi following multiple respiratory infections.

There may also be increased collapsibility or tracheomalacia of the trachea on forced expiration or during coughing (Figure 3).

\section{Tracheal stenosis}

\section{Congenital tracheal stenosis}

Congenital tracheal stenosis is a condition in which the tracheal cartilages are hypoplastic forming complete rings without a normal posterior membranous wall. The trachea is therefore rigid and non-distensible (11). This may occur diffusely throughout the trachea or in part of the trachea. On CT, the trachea is focally or completely narrowed with identifiable complete cartilaginous rings. There may be other associated congenital abnormalities including H-type tracheoesophageal fistula, laryngomalacia, subglottic stenosis, bronchial stenosis, hypoplasia or agenesis of the lungs.

\section{Post intubation injury/post traumatic Injury}

Tracheal stenosis can be encountered following tracheotomy and intubation $(12,13)$. It occurs primarily at two sites: (I) at the tracheostomy opening or stoma and (II) at the site of the balloon cuff. At the stoma site, the presence of a large stoma, superinfection or rigid connecting apparatus can lead to pressure erosion and subsequent strictures at the stoma. Granulation tissue and/or fibrosis often form on the anterior or lateral walls forming a triangular shaped area of narrowing. Calcium deposits can be seen in the tracheal wall on CT. Occasionally, there can be inversion of the anterior tracheal wall into the tracheal lumen forming an anterior 
wall flap from above the stoma. If the tracheostomy tube is removed, the flap may cause a ball-valve obstruction in the tracheal lumen.

A cuff stenosis characteristically occurs $1-2 \mathrm{~cm}$ below the stoma and has a characteristic smooth circumferential "hourglass" narrowing, typically $1-4 \mathrm{~cm}$ in length, that can be demonstrated on radiographs and CT. The length and severity of the stenosis is related to the size and shape of the cuff, cuff pressure, duration of intubation and peak inspiratory pressure. These factors may contribute to pressure necrosis from vascular compromise. Mucosal ulceration, chondritis and cartilaginous disruption may ensue leading to a stenosis from granulation tissue and fibrous tissue. When cartilaginous destruction is present a focal area of malacia may develop that can be demonstrated on inspiratory/expiratory CT imaging. In a small number of cases, an associated tracheoesophageal fistula may develop often due to prolonged intubation with a coexisting nasogastric tube (Figure 4).

Posttraumatic tracheal stenosis may occur following penetrating injuries, strangulation, or motor vehicle accidents in which there is healing following a partial tear that may have been initially missed or as a complication of a surgical airway anastomosis. A focal circumferential or "hourglass" stenosis is demonstrated by radiographs and CT (Figure 4).

\section{Idiopathic laryngotracheal stenosis}

Idiopathic laryngotracheal stenosis is a rare cause of laryngeal and tracheal narrowing that is typically found in middle aged women who do not have a history of prior trauma, infection or systemic disorder (14). On CT the appearance is variable but may include smooth, tapered, irregular, nodular or eccentric forms of narrowing typically measuring $2-4 \mathrm{~cm}$ in length.

\section{Saber sheath trachea}

Saber sheath trachea is an acquired condition that is seen in association with chronic obstructive lung diseases, particularly chronic bronchitis (15). Repeated excessive coughing with resultant tracheal collapse purportedly leads to degenerative softening, revascularization and ossification of the tracheal cartilages. In this condition, there is a diffuse "side-to-side" narrowing of the tracheal that is classically intrathoracic in distribution beginning at the thoracic inlet and extending to the distal trachea. The coronal narrowing and sagittal widening has a tracheal index (coronal/sagittal diameter) $<0.5$ at the level of the aortic arch. There is no associated tracheomalacia (Figure 4).

\section{Relapsing polychondritis}

Relapsing polychondritis is an uncommon autoimmune disorder that causes progressive, episodic inflammation and destruction of hyaline cartilage of the ears, nose, upper respiratory tract, and joints $(16,17)$. The destroyed cartilage is replaced by fibrosis and granulomatous tissue. The tracheal involvement is limited to the cartilage and perichondrium. Airway involvement may be seen in up to $50 \%$ of patients and is associated with recurrent pneumonia that is the most common cause of death. CT may demonstrate smooth diffuse thickening of the trachea and main bronchi, sometimes involving the segmental and subsegmental airways, typically sparing the membranous wall which is devoid of cartilage. The thickened airway wall may be calcified. In the acute inflammatory stage, expiratory CT may demonstrate tracheobronchomalacia with excessive collapse and associated air trapping in the lung. Once the destroyed cartilage is replaced by fibrosis a fixed stenosis is seen (Figure 5).

\section{Tracheopathia osteochondroplastica}

Tracheopathia osteochondroplastica is an uncommon benign disorder in which multiple firm osteocartilaginous nodules form in the submucosa of the anterior and lateral walls of the cervical and intrathoracic trachea and main bronchi, typically sparing the posterior membranous wall $(18,19)$. It is most common in men older than 50 years of age. The size and distribution of the nodules is best demonstrated by CT. Nodules may range in size from a few millimeters to larger obstructing nodules and may demonstrate calcification on CT. The tracheal wall is typically rigid on inspiratory and expiratory imaging (Figure 5).

\section{Amyloidosis}

Amyloidosis is an uncommon disorder in which there is extracellular deposition of insoluble proteinaceous fibrils that stain with Congo red (20-22). The fibrils disrupt the normal architecture of the airway by forming focal or diffuse thickening of the airway wall or nodules and masses that typically grow slowly over time and may cause airway 

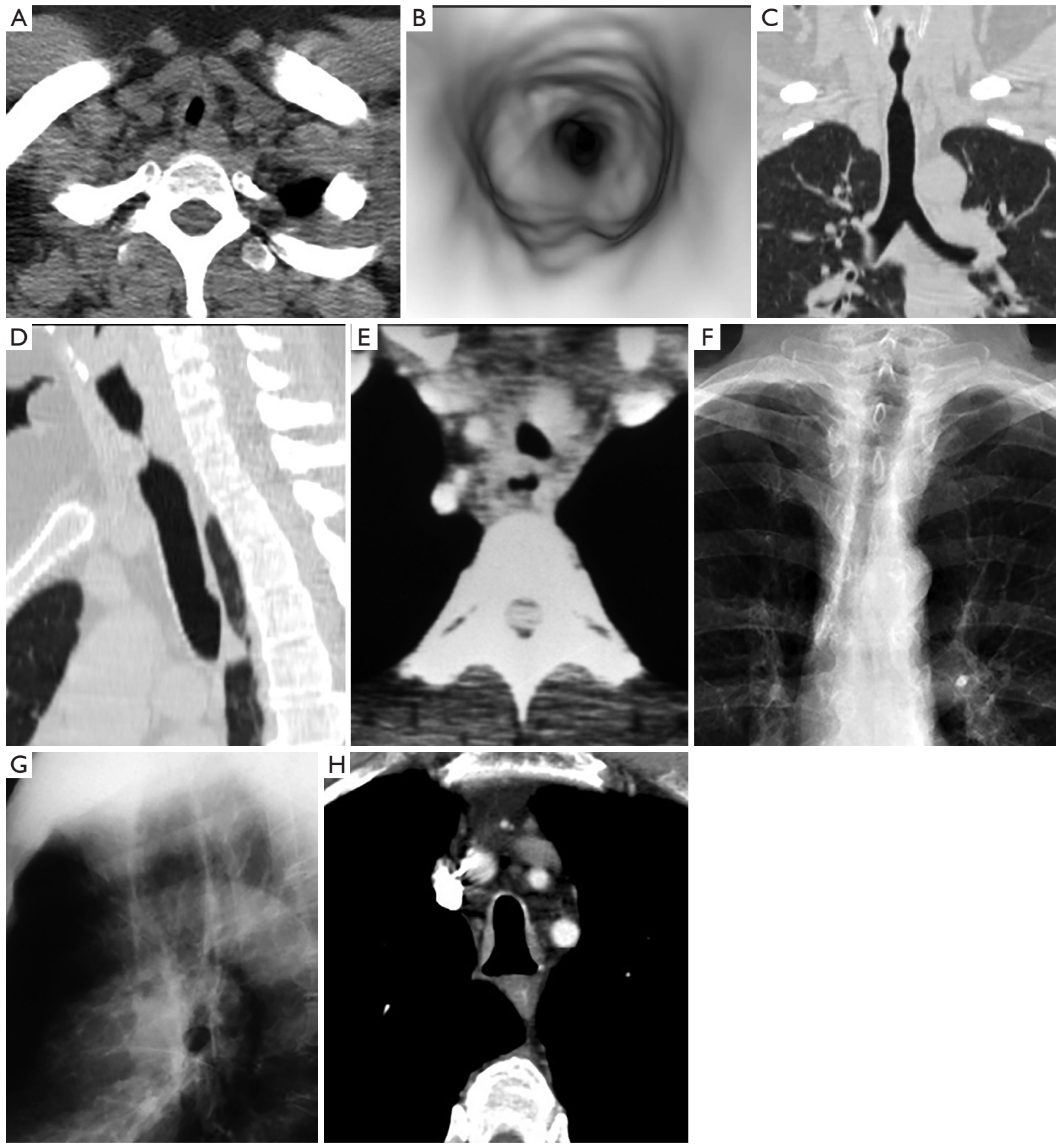

Figure 4 Tracheal stenosis. In a patient with post intubation "cuff stenosis" (A-D), an axial CT image (A) demonstrates circumferential thickening of the tracheal wall and narrowing of the lumen. A virtual bronchoscopic image (B) shows the circumferential stenosis. Coronal (C) and sagittal (D) CT images reveal the typical "hour glass" stenosis in the trachea at the level of the endotracheal tube cuff. In a patient with post traumatic tracheal stenosis (E), an axial CT image (E) of the proximal thoracic trachea reveals a circumferential stenosis of the trachea with diffuse irregular soft tissue thickening due to fibrosis. The enhancing right innominate artery forms the anterior wall of the trachea in this patient who presented with a delayed tracheal stenosis following a missed tracheal laceration resulting from trauma. In a patient with a "saber sheath trachea" (F-H), a posterior anterior $(\mathrm{PA})(\mathrm{F})$ and lateral $(\mathrm{G})$ chest radiographs and axial CT images (H) demonstrate diffuse narrowing the intrathoracic trachea. Note that the trachea is narrowed transversely and elongated in the sagittal plane and that the tracheal narrowing begins in the proximal thoracic trachea below the thoracic inlet. 

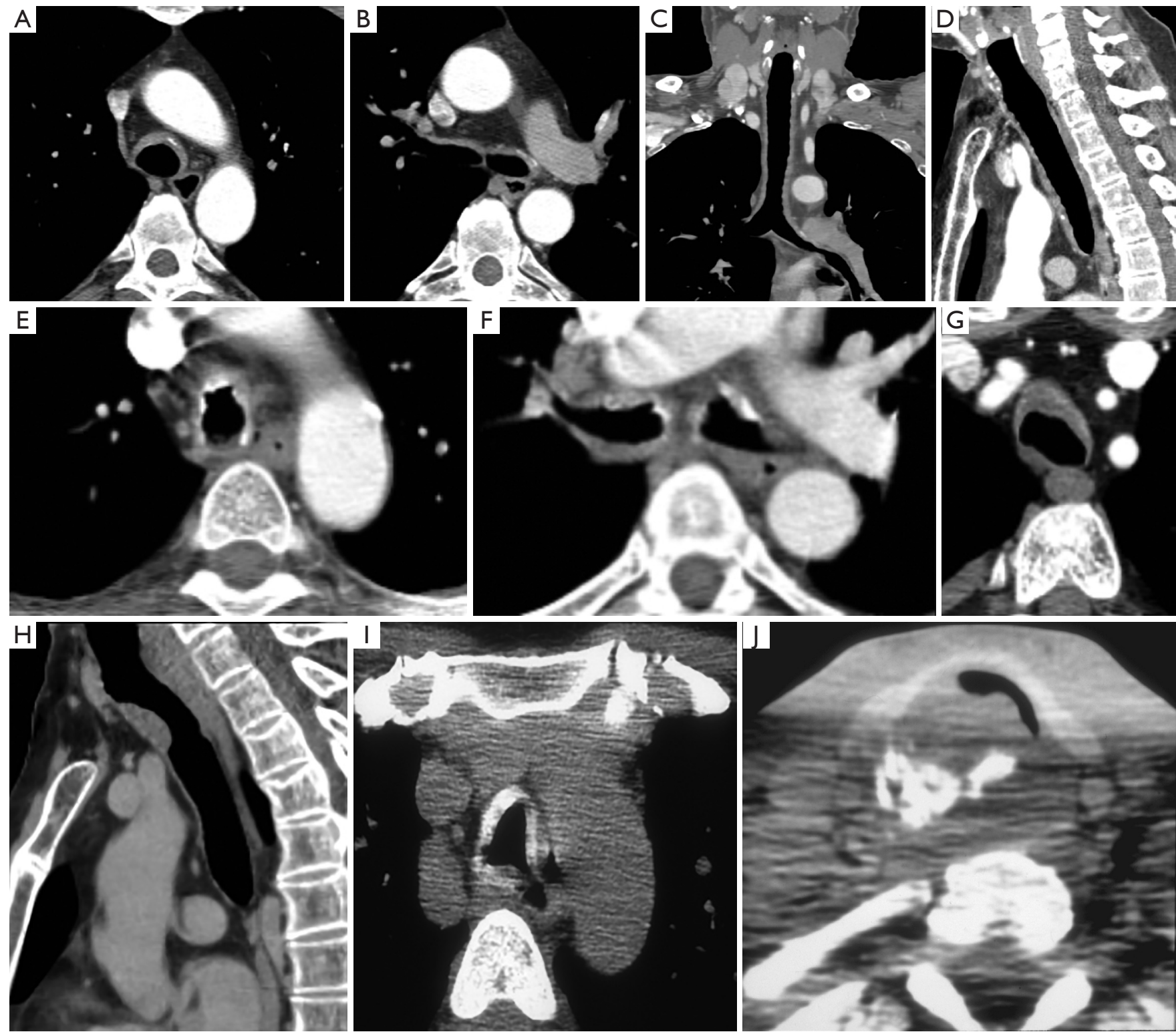

Figure 5 Diffuse tracheal stenosis. In a patient with relapsing polychondritis (A-D), axial CT images of the thoracic trachea (A) and main bronchi (B) and coronal (C) and sagittal (D) images of the trachea demonstrate diffuse thickening of the cartilaginous portions of the trachea, sparing the posterior membranous wall. There are scattered calcifications within the abnormally thickened tracheal wall. In a patient with tracheopathia osteochondroplastica (E,F), axial CT of the trachea (E) and main bronchi (F) show smooth nodular thickening of the anterior and lateral walls of the trachea and main bronchi in which cartilaginous calcification is seen. The posterior membranous wall is spared. In a patient with amyloidosis $(\mathrm{G}, \mathrm{H})$, axial $(\mathrm{G})$ and sagittal $(\mathrm{H}) \mathrm{CT}$ images in soft tissue window reveal a circumferential stenosis of the thoracic trachea that involves the cartilaginous and membranous wall of the trachea. In two additional patients with amyloidosis (I,J), axial CT images demonstrate calcification within tracheal amyloid. In image (I) the tracheal wall is diffusely thickened and calcified involving the membranous and cartilaginous walls. In a different patient with tracheal amyloid (J), there is a large calcified soft tissue mass that nearly obstructs the cervical trachea. 
obstruction. The deposits may calcify and may enhance with iodinated CT contrast or gadolinium on MRI exams. There may be associated hilar and mediastinal lymphadenopathy with may contain calcifications (56 urban) (Figure 5).

\section{Granulomatosis with polyangiitis (GPA)}

GPA formerly known as Wegener's granulomatosis, is an autoimmune systemic vasculitis in which tracheobronchial involvement occurs in up to $50 \%$ of patients $(23,24)$. Concentric tracheal narrowing may be focal or diffuse and occurs most commonly in the subglottic region. Associated destruction of the tracheal cartilage or cricoid cartilage is less common. Involvement of the large airways results in smooth or nodular tracheobronchial wall thickening which may progress to focal or diffuse stenosis. The posterior membranous wall is not typically spared as the case in relapsing polychondritis. Tracheomalacia is not an associated finding (Figure 6).

\section{Fibrosing mediastinitis}

Fibrosing mediastinitis is a complication of granulomatous mediastinitis most often resulting from infection from Histoplasma capsulatum and less often Mycobacterium tuberculosis $(25,26)$. It is characterized by a proliferation of fibrous tissue in the mediastinum that may surround, invade or obliterate mediastinal structures including the trachea and bronchi, esophagus, pulmonary arteries, veins and thoracic duct. On CT calcification is often seen within the infiltrating fibrous tissue in the mediastinum. MRI is useful to evaluate vascular invasion (Figure 6).

\section{Tuberculosis}

Tuberculous tracheal stenosis may result from extrinsic compression from hyperplastic lymph nodes or by granulomatous changes in the tracheal wall. Infection may develop from direct contact of infected secretions particularly when parenchymal cavitary lesions are present or by submucosal spread through the lymphatics. In the acute hyperplastic stage, ulceration and necrosis of the wall develops as tubercles form in the submucosal layer. Over time fibrosis develops leading to a tracheal and/ or bronchial stenosis (27). On CT, the hyperplastic stage manifests as diffuse irregular tracheal bronchial luminal narrowing. There may be associated hilar or mediastinal lymphadenopathy that may demonstrate peripheral enhancement and central necrosis and/or cavitary lesions and tree-in-bud opacities in the infected lungs. Once healing occurs the airway develops a smooth stenotic appearance and there may be residual bronchiectasis in the affected lung. If the stenosis is obstructing, distal atelectasis may occur. Lymph nodes often develop calcification over time. As a late sequelae, erosion of a lymph node into a bronchus may form a broncholith (Figure 6).

\section{Benign neoplasms}

\section{Tracheobronchial papillomatosis}

Tracheobronchial papillomatosis is generally acquired at birth through vaginal delivery in a mother infected with human papilloma virus. Children and young adults may present with laryngeal papillomatosis and in about $5 \%$ of affected patients dissemination will spread down into the trachea into the smaller airways and in $1 \%$ of patients the lungs will be affected. Malignant transformation into squamous cell carcinoma can occur in up to $2 \%$ of affected patients. On CT imaging, tracheobronchial papillomas appear as nodular intraluminal filling defects in the trachea and bronchi. When lung involvement is present small nodules or cysts are seen usually in the dependent portions of the lower lobes. Nodules may cavitate and some may develop air-fluid levels. When malignant degeneration occurs, the nodules will enlarge in size and may have associated lymphadenopathy and bronchial obstruction with associated lobar collapse (Figure 6).

\section{Squamous cell papilloma}

Squamous cell papilloma is the most common benign tumor of the trachea in adults and is associated with smoking. It occurs more frequently in men than women and usually manifests on CT as a solitary intraluminal soft tissue nodule without invasive features (28).

\section{Hamartoma}

Hamartoma is the second most common benign tracheal tumor. It presents as focal smooth nodular endoluminal filling defect and can be distinguished from other benign mesenchymal tumors on CT by the presence of fat and cartilaginous calcification, present in $25 \%$ of patients. Hamartomas characteristically grow slowly and do not 

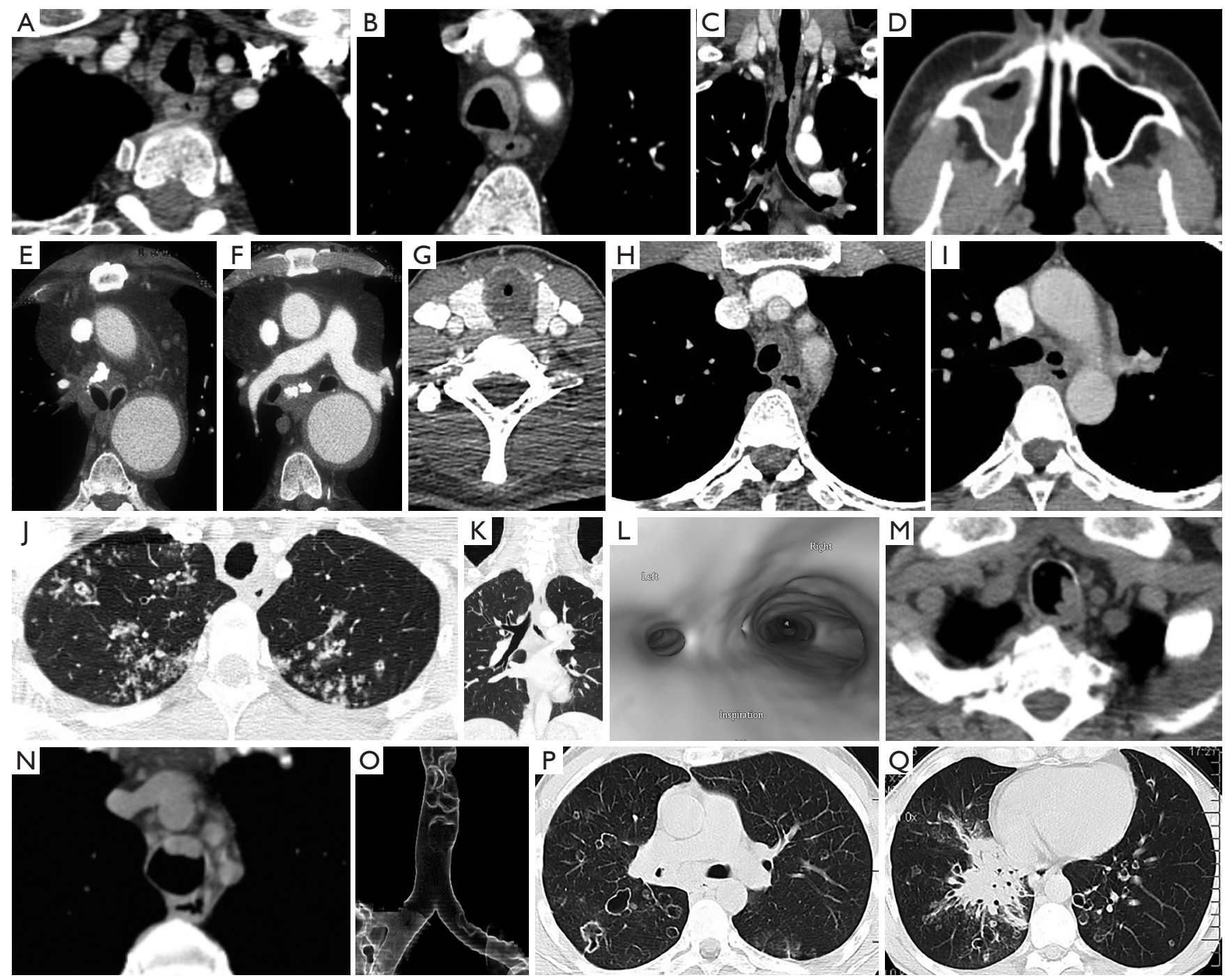

Figure 6 Inflammatory and infectious tracheal lesions. In a patient with granulomatous polyangiitis (GPA) (A-D), axial CT images of the proximal trachea (A) and mid thoracic trachea (B), and coronal image of the trachea (C) demonstrate smooth circumferential soft tissue thickening of the trachea with stenosis of the lumen. A CT image of the sinuses (D) reveals diffuse thickening of the maxillary antrum. In a patient with fibrosing mediastinitis $(\mathrm{E}, \mathrm{F})$, axial CT images in soft tissue windows of the carina $(\mathrm{E})$ and main bronchi $(\mathrm{F})$ demonstrate narrowing and distortion of the carina and main bronchi by surrounding calcified soft tissue representing fibrosis. There is also narrowing of the right interlobar pulmonary artery by the fibrosis $(\mathrm{F})$. In a patient with tuberculous tracheobronchial stenosis, hyperplastic phase (G-J), axial CT images of the distal cervical trachea (G), mid thoracic trachea (H) and main bronchi (I) in soft tissue windows reveals diffuse irregular thickening of the tracheal wall and diffuse stenosis of the trachea and left main bronchus in this patient with a chronic history of active post-primary tuberculosis. An axial CT image in lung window (J) reveals tree-in-bud opacities, bronchiectasis and bronchial wall thickening consistent with active tuberculosis. In a patient with a tuberculous tracheobronchial stenosis, fibrotic phase (K,L), a coronal CT $(\mathrm{K})$ in lung windows and a virtual bronchoscopic image $(\mathrm{L})$ demonstrate a smooth fibrotic stenosis of the left main bronchus from healed tuberculosis. In a patient with tracheobronchial papillomatosis (M-O), axial CT images of the proximal $(\mathrm{M})$ and mid $(\mathrm{N})$ thoracic trachea reveals multiple nodular soft tissue filling defects without evidence for invasion. A 3D-rendering of the trachea and main bronchi (O) demonstrates multiple nodules in the trachea and left main bronchus. In a separate patient with tracheobronchial papillomatosis (P,Q), axial CT images through the lower lobes in lung windows $(\mathrm{P})$ and $(\mathrm{Q})$ reveals multiple thin-walled pulmonary cysts in the lower lungs. There is a spiculated right lower lobe mass representing a squamous cell carcinoma that nearly occludes the bronchus intermedius $(\mathrm{P})$ and right lower lobe bronchus (Q). 
exhibit invasive features on imaging. Distal atelectasis or pneumonia may present when hamartomas develop in bronchi.

\section{Chondroma}

Chondromas are uncommon benign cartilaginous tumors that occur most frequently in middle aged men. The most common location is in the inner surface of the cricoid lamina (70\%). They occur in the tracheal less commonly (29). On CT, the tumor presents as a well-defined mass, frequently contains mottled cartilaginous calcification and is indistinguishable from a chondrosarcoma (Figure 7).

\section{Malignant tracheal neoplasms}

Most tumors involving the trachea tend to be malignant (30-32). Direct invasion of the trachea by adjacent malignancies is most common and may arise from cancers of the larynx, thyroid, esophagus and lung. Tracheal malignancies from hematogenous metastases arising from melanoma, breast, colorectal, renal and primary tracheal malignancies are less common (Figure 7). Squamous cell carcinoma and adenoid cystic carcinoma of the trachea are the most common primary tracheal malignancies. Other primary tracheal malignancies including mucoepidermoid carcinoma, carcinoid tumors, chondrosarcomas and lymphomas are less common.

\section{Squamous cell cancer}

Squamous cell cancers are the most common primary malignancy of the trachea and are smoking related tumors, occurring most commonly in males 50-60 years old. Patients may have synchronous or metachronous squamous cancers of the head and neck or lungs. Squamous cell cancers may arise in any part of the trachea or bronchi but most commonly occur in the posterior wall of the distal two thirds of the trachea. On CT, the tumors generally present as a polypoid intraluminal mass with smooth, lobular or irregular margins or as focal circumferential thickening of the trachea. Approximately one third of tumors present with associated metastatic lymphadenopathy and/or lung metastases. There may be adjacent invasion of the mediastinal structures including the esophagus which may lead to tracheoesophageal or bronchoesophageal fistula. On FDG PET, the tracheal tumor and lymphadenopathy demonstrate increased FDG uptake due to high metabolic activity of the tumor (Figure 7).

\section{Adenoid cystic carcinoma}

Adenoid cystic carcinoma is the second most common primary malignancy of the trachea, presents in the fourth decade of life, affecting men and women equally and is not smoking related. Adenoid cystic carcinoma may present as circumferential or diffuse thickening of the trachea or as a focal endoluminal polypoid nodule or mass. The lower trachea and main bronchi are most commonly affected. The tumor has a propensity to growth by endophytic spread in the submucosal plane of the trachea. Cross-sectional imaging may reveal extension into the adjacent mediastinal fat. In $10 \%$ of patients, metastases may occur in regional lymph nodes. Hematogenous metastases may occur in lung, bones or liver late in the progression of the disease. These tumors generally demonstrate slow growth, thus FDG uptake on PET/CT is highly variable (Figure 7).

\section{Mucoepidermoid carcinoma}

Mucoepidermoid tumors are very rare tumors of the trachea and main bronchi and may be of low-grade or high-grade malignancy. On imaging, they present as a focal soft tissue endoluminal mass without distinguishing features from other airway malignancies (Figure 7).

\section{Tracheomalacia}

Tracheomalacia refers to weakness of the tracheal wall with resultant collapsibility of the trachea (33-35). It may be due to a primary immaturity or a secondary softening or destruction of the cartilaginous rings or to excessive flaccidity of the posterior membranous wall. There are many known causes of tracheomalacia including congenital absence or hypoplasia of cartilaginous rings, traumatic causes including mechanical ventilation with cuffed endotracheal tubes or blunt trauma, inflammatory diseases such as relapsing polychondritis and compression by adjacent masses such as goiters and aberrant vessels or adjacent aneurysms. Tracheomalacia may also be idiopathic.

Dynamic CT imaging or inspiratory and expiratory CT imaging are the desired imaging procedures for evaluating tracheobronchomalacia. Expiration or coughing will normally result in narrowing of the tracheobronchial lumen by $10-30 \%$ with normal invagination of the posterior membranous wall (34). In tracheomalacia, the trachea will 

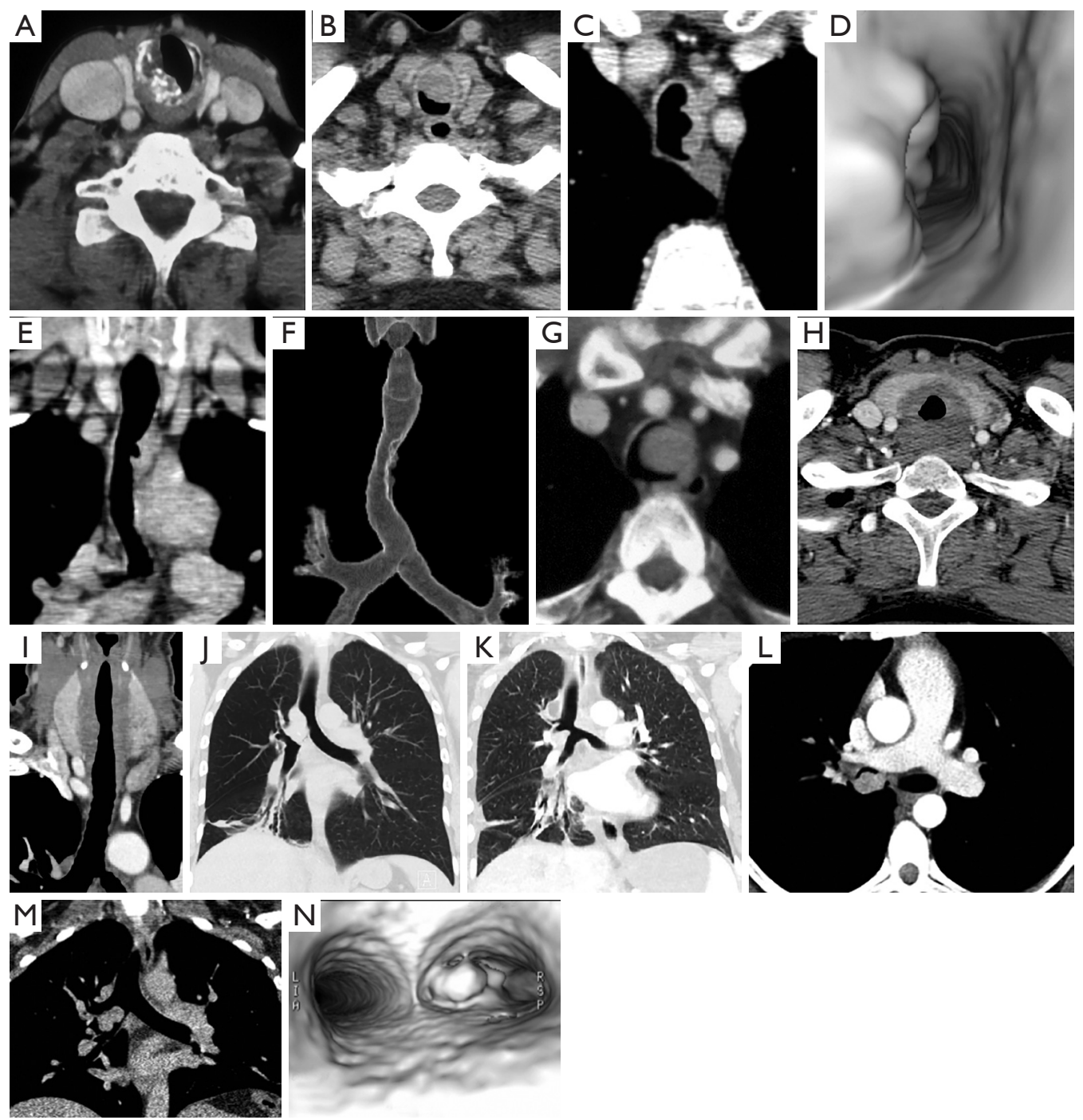

Figure 7 Tracheal tumors. In a patient with a chondroma of the cricoid cartilage (A), axial CT image (A) demonstrates a nodular tumor arising from the right aspect of the cricoid cartilage that contains dense calcification typical of a cartilaginous tumor. In a patient with a tracheal metastasis from melanoma (B), an axial CT image (B) at the thoracic inlet reveals a soft tissue mass arising from the anterior tracheal wall causing significant narrowing of the tracheal lumen. In a patient with squamous cell carcinoma of the trachea (C-F), an axial CT image (C) and virtual bronchoscopic image (D) of the mid trachea reveals a spiculated and nodular broad-based tumor arising from the left lateral wall of the trachea with associated thickening of the tracheal wall. There is an adjacent prominent lymph node is the left paratracheal region (C). A coronal CT image (E) and 3D surface rendering (F) of the trachea demonstrate the focal mass along the left lateral wall of the trachea. In a patient with adenoid cystic carcinoma of the trachea $(G)$, an axial CT $(G)$ in soft tissue window shows a soft tissue nodule arising from the left lateral tracheal wall that protrudes into the tracheal lumen. Notice the thickening of the left anterolateral tracheal wall by the tumor. In a different patient with adenoid cystic carcinoma of the trachea $(\mathrm{H}, \mathrm{I})$, axial $(\mathrm{H})$ and coronal $(\mathrm{I})$ soft tissue images of the trachea reveal diffuse smooth thickening of the trachea extending from the subglottic region to the thoracic inlet typical of diffuse submucosal spread of the tumor. In another patient with adenoid cystic carcinoma (J,K), coronal CT image in lung window demonstrates the pre-operative appearance (J) of an adenoid cystic carcinoma of the distal trachea and right main bronchus with distal atelectasis in the right lower lobe. A coronal CT image reveals the expected post-operative appearance following a carinal resection $(\mathrm{K})$ in which the distal trachea and proximal main bronchi were resected. The right upper lobe bronchus was anastomosed to the trachea (end-to-side), the distal trachea was anastomosed to the left main bronchus (end-to-end) and the bronchus intermedius was anastomosed to the left main bronchus (end-to-side). In a patient with mucoepidermoid tumor at the carina (L-N), axial CT (L), coronal CT (M) in soft tissue windows and virtual bronchoscopic (N) image reveal a nodular soft tissue tumor obstructing the proximal right main bronchus extending to the carina. 

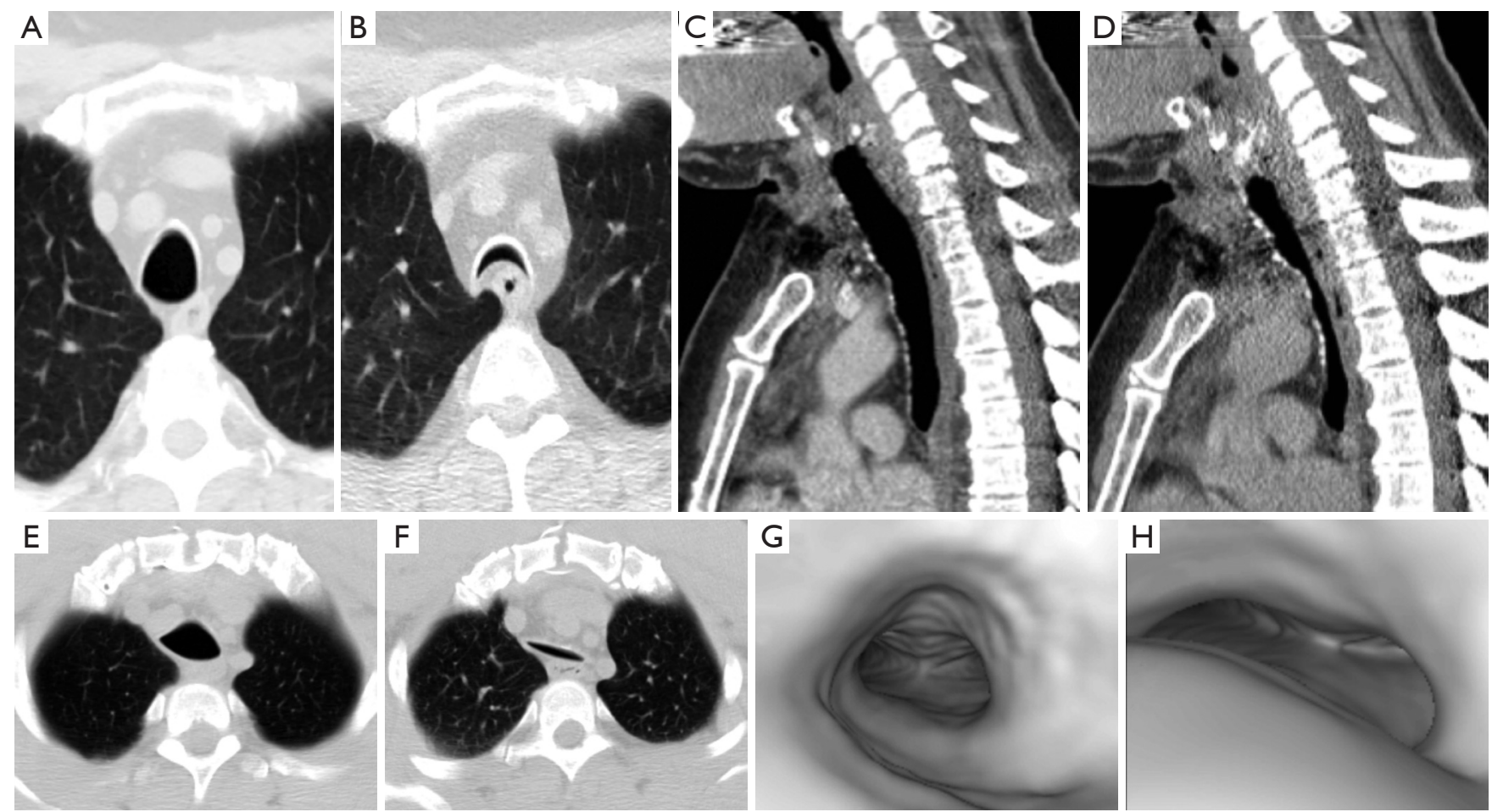

Figure 8 Tracheomalacia. In a patient with tracheomalacia (A-D), axial CT images through the proximal intrathoracic trachea on inspiration (A) and expiration (B) demonstrates $>70 \%$ narrowing of the tracheal lumen on expiration. Note that on expiration (B) there is marked anterior deviation of the posterior membranous wall of the trachea demonstrating the "frown sign". Sagittal CT images through the trachea on inspiration (C) and expiration (D) reveals marked collapse of the intrathoracic trachea on expiration. In another patient with tracheomalacia (E-H), axial CT on inspiration (E) and expiration (F) reveal an elongated posterior membranous wall of the trachea and $>70 \%$ collapse on expiration (F). Virtual bronchoscopic images on inspiration (G) and expiration (H) demonstrate significant collapse of the trachea consistent with tracheomalacia.

decrease in caliber by more than $70 \%$ on forced expiratory imaging or coughing. Cross sectional imaging on CT will demonstrate the "frown sign" in which the posterior membranous wall becomes concave in shape approximating the anterior wall of the trachea (Figure 8).

\section{Conclusions}

CT is the imaging modality of choice for evaluation of tracheal abnormalities. A host of benign and malignant diseases may affect the trachea primarily and secondarily. Familiarity with their imaging appearances will enhance diagnostic evaluation of the trachea.

\section{Acknowledgements}

None.

\section{Footnote}

Conflicts of Interest: The authors have no conflicts of interest to declare.

\section{References}

1. Heidinger BH, Occhipinti M, Eisenberg RL, et al. Imaging of large airways disorders. AJR Am J Roentgenol 2015;205:41-56.

2. Boiselle PM. Imaging of the large airways. Clin Chest Med 2008;29:181-93.

3. Javidan-Nejad C. MDCT of trachea and main bronchi. Radiol Clin North Am 2010;48:157-76.

4. Lee KS, Boiselle PM. Update on multidetector computed tomography imaging of the airways. J Thorac Imaging 2010;25:112-4.

5. Ferretti GR, Bithigoffer C, Righini CA, et al. Imaging of 
tumors of the trachea and central bronchi. Thorac Surg Clin 2010;20:31-45.

6. Kang EY. Large airway diseases. J Thorac Imaging 2011;26:249-62.

7. Siegel MJ, Shakelford GD, Francis RS, et al. Tracheal bronchus. Radiology 1979;130:353-5.

8. Goo JM, Im JG, Ahn JM, et al. Right paratracheal air cysts in the thoracic inlet: clinical and radiologic significance. AJR Am J Roentgenol 1999;173:65-70.

9. Shin MS, Jackson RM, Ho KJ. Tracheobronchomegaly (Mounier-Kuhn syndrome): CT diagnosis. AJR Am J Roentgenol 1988;150:777-9.

10. Dunne MG, Reiner B. CT features of tracheobronchomegaly. J Comput Assist Tomogr 1988;12:388-91.

11. Benjamin B, Pitkin J, Cohen D. Congenital tracheal stenosis. Ann Otol Rhinol Laryngol 1981;90:364-71.

12. Cooper JD, Grillo. The evolution of tracheal injury due to ventilator assistance through cuffed tube. A pathologic study. Ann surg 1969;169:334.

13. Wood DE, Mathisen D. Late complications of tracheotomy. Clin Chest Med 1991;12:597-609.

14. Bhalla M, Grillo HC, McLoud TC, et al. Idiopathic laryngotracheal stenosis: radiologic findings. AJR Am J Roentgenol 1993;161:515-7.

15. Greene R. Saber-sheath trachea: relation to chronic obstructive pulmonary disease. AJR Am J Roentgenol 1978; $130: 441-5$

16. Im JG, Chung JW, Han SK, et al. CT manifestations of tracheobronchial involvement in relapsing polychondritis. J Comput Assist Tomogr 1988;12:792-3.

17. McAdam LP, O'Hanlan MA, Bluestone R, et al. Relapsing polychondritis: prospective study of 23 patients and a review of the literature. Medicine (Baltimore) 1976;55:193-215.

18. Mariotta S, Pallone G, Pedicelli G, et al. spiral CT and endoscopic findings in a case of tracheobronchopathia osteochondroplastica. J Comput Assist Tomogr 1997;21:418-20.

19. Onitsuka H, Hirose N, Watanabe K. Computed tomography of tracheopathia osteoplastica. AJR Am J Roentgenol 1983;140:268-70.

20. Pickford HA, Swenson SJ, Utz JP. Thoracic crosssectional imaging of amyloidosis. AJR Am J Roentgenol 1997;168:351-5.

21. Cordier JF, Loire R, Breene J. Amyloidosis of the lower respiratory tract. Chest 1986;90:827-31.

22. Urban BA, Fishman EK, Goldman SM, et al. CT evaluation of amyloidosis: spectrum of disease. Radiographics 1993;13:1295-308.

23. Screaton NJ, Sivasothy P, Flower CD, et al. Tracheal involvement in Wegener's granulomatosis: evaluation using spiral CT. Clin Radiol 1998;53:809-15.

24. Stein MG, Gamsu G, Webb WR, et al. Computed tomography of diffuse tracheal stenosis in Wegener granulomatosis. J Comput Assist Tomogr 1986;10:868-70.

25. Farmer DW, Moore E, Amparo E, et al. Calcific fibrosing mediastinitis: demonstration of pulmonary vascular obstruction by magnetic resonance imaging. AJR Am J Roentgenol 1984;143:1189-91.

26. Weinstein JB, Aronberg DJ, Sagel SS. CT of fibrosing mediastinitis: findings and their utility. AJR Am J Roentgenol 1983;141:247-51.

27. Choe, KO Jeong HJ, Sohn HY. Tuberculous bronchial stenosis: CT findings in 28 cases. AJR Am J Roentgenol 1990;155:971-6.

28. Aylward TD, Glege JB Jr. Primary papilloma of the trachea. Ann Thorac Surg 1973;16:620-3.

29. Hyams VJ, Rabuzzu DD. Cartilaginous tumors of the larynx. Laryngoscope 1970;80:755-67

30. Ngo AV, Walker CM, Chung JH, et al. Tumors and tumorlike conditions of the large airways. AJR Am J Roentgenol 2013;201:301-13.

31. Park CM, Goo JM, Lee HJ, et al. Tumors in the tracheobronchial tree: CT and FDG PET features. Radiographics 2009;29:55-71.

32. Wu CC, Shepard JA. Tracheal and airway neoplasms. Semin Roentgenol 2013;48:354-64.

33. Boiselle PM, Feller-Kopman D, Ashiku S, et al. Tracheobronchomalacia: Evolving role of dynamic multislice helical CT. Radiol Clin N Am 2003;41:627-36

34. Stern EJ, Graham CM, Webb WR, et al. Normal trachea during forced expiration: dynamic CT measurements. Radiology 1993;187:27-31.

35. Aquino SL, Shepard JA, Ginns LC, et al. Acquired tracheomalacia: detection by expiratory CT scan. J Comput Assist Tomogr 2001;25:394-9.

Cite this article as: Shepard JO, Flores EJ, Abbott GF. Imaging of the trachea. Ann Cardiothorac Surg 2018;7(2):197209. doi: $10.21037 /$ acs.2018.03.09 